Supporting Information

\title{
Mass spectrometric quantitation of apurinic/apyrimidinic sites in tissue DNA of rats exposed to tobacco-specific nitrosamines and in lung and leukocyte DNA of cigarette smokers and nonsmokers
}

Jiehong Guo*, Haoqing Chen, Pramod Upadhyaya, Yingchun Zhao, Robert J. Turesky, and Stephen S. Hecht

Masonic Cancer Center, University of Minnesota

\section{Table of Contents}

(2 schemes, 9 Tables, 5 Figures, references, 15 pages)

Scheme S1. Derivatization reaction of the ring-opened aldehydic form of the deoxyribosyl moiety with PMOA

Scheme S2. The formation of an AP site and POB-DNA adduct N7POBG from NNKOAc

Table S1. Levels of AP sites in human lung DNA and subject information

Table S2. Levels of AP sites in human leukocyte DNA and subject information

Table S3. AP site levels in control rat lung analyzed along with human samples

Table S4. dG levels in rat tissues

Table S5a. Comparison of POB-DNA adduct levels from NNK acute exposures

Table S5b. Comparison of POB-DNA adduct levels from NNK chronic exposures

Table S6. Comparison of POB-DNA adduct levels from (S)-NNN exposures

Table S7. Comparison of DNA adducts and AP sites in cigarette smokers and nonsmokers

Table S8. AP site levels in human leukocyte DNA based on subject smoking status, gender, race and age

Figure S1. Flow chart of the experimental procedure for analysis of AP sites and DNA adducts

Figure S2. HPLC-ESI-MS/MS chromatograms of PMOA-dR and POB-DNA adducts

Figure S3. Dependence of increased AP site formation on $\mathrm{N}^{7} \mathrm{POBG}$ or $\mathrm{O}^{2} \mathrm{POBdT}$ in lung and liver of rats treated with $5.2 \mathrm{mg} / \mathrm{kg}$ and $20.7 \mathrm{mg} / \mathrm{kg} \mathrm{NNK}$ for 4 days

Figure S4. Dependence of increased AP site formation on $\mathrm{N}^{7} \mathrm{POBG}$ or $\mathrm{O}^{2} \mathrm{POBdT}$ and dependence of $\mathrm{O}^{2} \mathrm{POBdT}$ on $\mathrm{N}^{7} \mathrm{POBG}$ in the liver of rats treated with $5 \mathrm{ppm}$ NNK in drinking water for 70 weeks

Figure S5. Box and whisker plots of AP site formation in human lung and leukocyte DNA.

References 


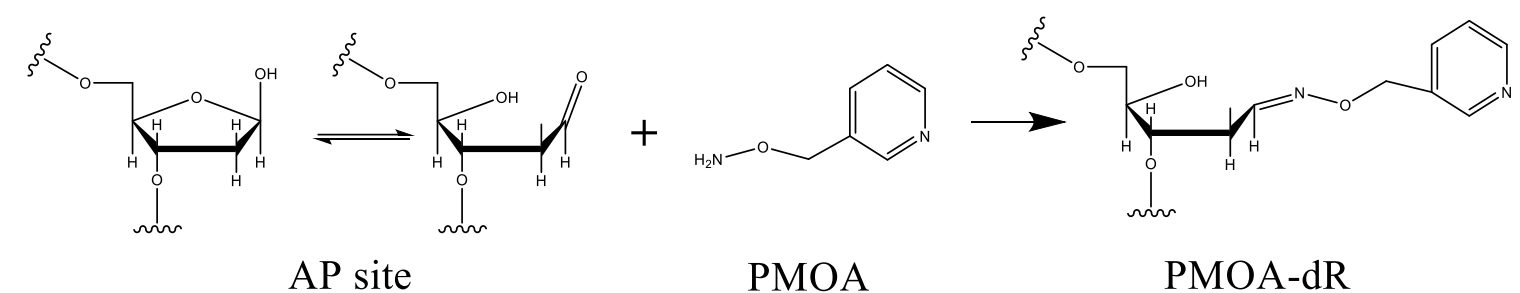

Scheme S1. Derivatization reaction of the ring-opened aldehydic form of the deoxyribosyl moiety with PMOA forms a stable hydrazone for LC-MS/MS analysis.

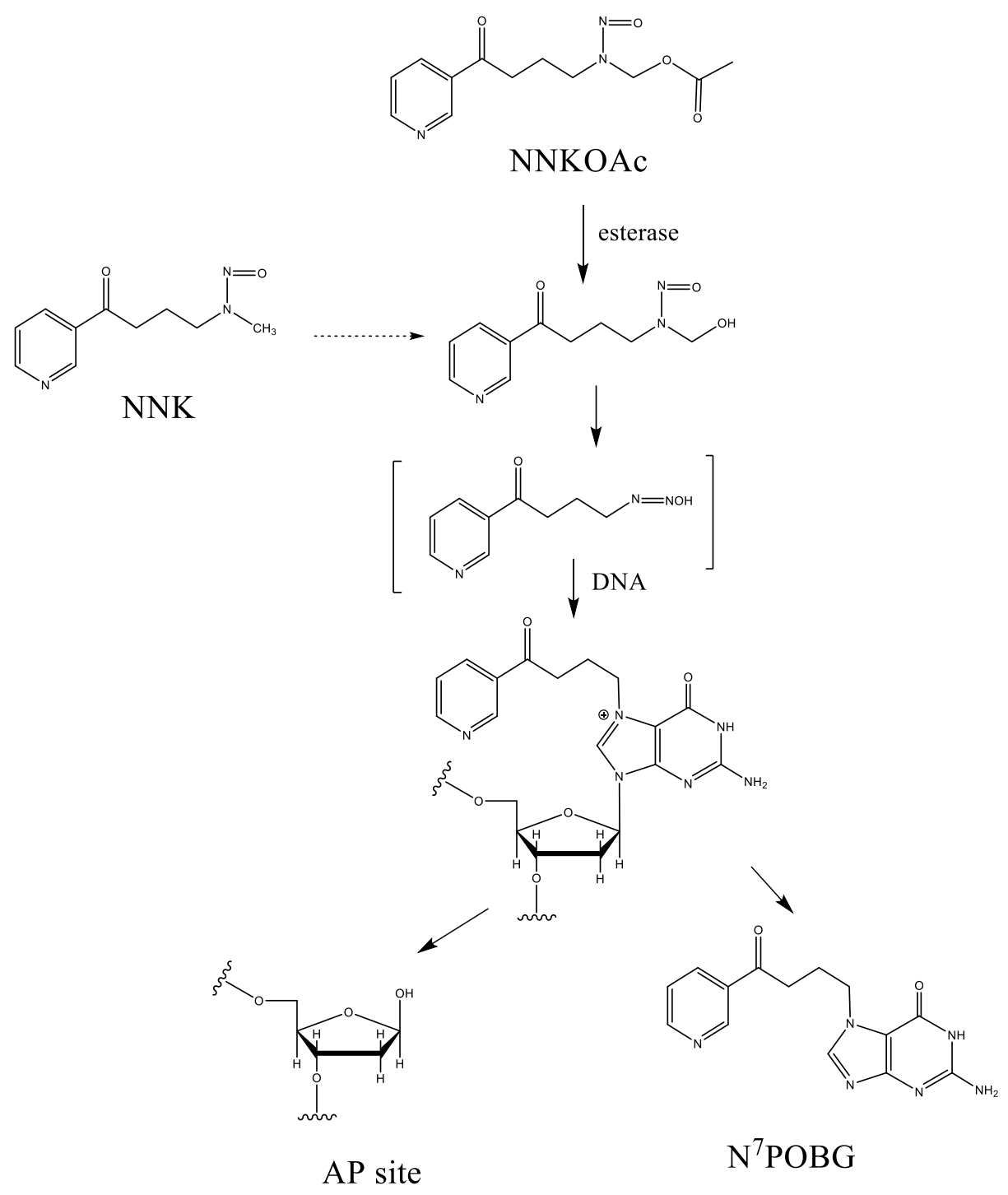

Scheme S2. The formation of an AP site and POB adduct N ${ }^{7} P O B G$ from NNKOAc 
Table S1. Levels of AP sites (PMOA-dR) in human lung DNA and subject information

\begin{tabular}{|c|c|c|c|c|c|c|c|c|}
\hline Subject & $\begin{array}{l}\text { Smoking } \\
\text { status }\end{array}$ & Gender & Race & $\begin{array}{l}\text { Age at } \\
\text { Surgery }\end{array}$ & $\mathrm{dG}(\mu \mathrm{mol})$ & $\begin{array}{l}\text { PMOA-dR } \\
\text { (per } 10^{7} \text { nts) }\end{array}$ & $\begin{array}{l}\text { Cotinine } \\
\text { (ng/mL) }\end{array}$ & $\begin{array}{c}\mathrm{NNAL} \\
\text { (pmol/mL) }\end{array}$ \\
\hline 1 & Nonsmoker & Female & White & 80 & 0.12 & 1.77 & $<3$ & \\
\hline 2 & Nonsmoker & Female & White & 49 & 0.11 & 1.86 & $<3$ & \\
\hline 3 & Nonsmoker & Female & White & & 0.21 & 1.46 & $<3$ & \\
\hline 4 & Nonsmoker & Female & White & & 0.10 & 1.86 & $<3$ & \\
\hline 5 & Nonsmoker & Male & White & 69 & 0.15 & 2.70 & $<3$ & \\
\hline 6 & Nonsmoker & Male & White & 56 & 0.22 & 2.24 & $<3$ & \\
\hline 7 & Nonsmoker & Male & White & $N A^{b}$ & 0.09 & 1.88 & $<3$ & \\
\hline 8 & Nonsmoker & Male & White & & 0.11 & 2.31 & $<3$ & \\
\hline 9 & Nonsmoker & Female & White & 74 & 0.17 & 3.54 & $<3$ & \\
\hline 10 & Nonsmoker & Female & White & 66 & 0.15 & 2.30 & $<3$ & \\
\hline 11 & Nonsmoker & Female & White & & 0.08 & 3.72 & $<3$ & \\
\hline 12 & Nonsmoker & Female & White & 66 & 0.12 & 1.82 & $<3$ & \\
\hline 13 & Nonsmoker & Male & White & 65 & 0.13 & 4.78 & $N A^{b}$ & \\
\hline 14 & Nonsmoker & Male & White & & 0.13 & 1.90 & $<3$ & \\
\hline 15 & Nonsmoker & Male & White & 62 & 0.21 & 6.40 & $<3$ & \\
\hline 16 & Nonsmoker & Male & White & 76 & 0.15 & 2.01 & $<3$ & \\
\hline 17 & Nonsmoker & Male & White & & 0.17 & 2.49 & 7 & \\
\hline \multicolumn{3}{|c|}{ Nonsmoker average $\pm \mathrm{SD}^{\mathrm{a}}$} & & $66 \pm 9$ & $0.14 \pm 0.04$ & $2.65 \pm 1.29$ & & \\
\hline 18 & Smoker & Female & White & 57 & 0.14 & 2.07 & 137 & \\
\hline 19 & Smoker & Female & White & 45 & 0.14 & 2.08 & 1244 & \\
\hline 20 & Smoker & Female & Black & 78 & 0.09 & 2.49 & 1099 & \\
\hline 21 & Smoker & Female & White & 55 & 0.15 & 2.21 & 855 & 0.92 \\
\hline 22 & Smoker & Female & White & 68 & 0.13 & 1.91 & 998 & \\
\hline 23 & Smoker & Female & White & & 0.06 & 2.18 & 1518 & \\
\hline 24 & Smoker & Female & White & & 0.09 & 2.59 & 1561 & \\
\hline 25 & Smoker & Female & White & 59 & 0.16 & 3.30 & 6363 & \\
\hline 26 & Smoker & Male & White & 56 & 0.11 & 6.58 & $N A^{b}$ & \\
\hline 27 & Smoker & Male & Black & 55 & 0.15 & 1.96 & 4372 & \\
\hline 28 & Smoker & Male & White & 64 & 0.13 & 2.06 & 3955 & 3.7 \\
\hline 29 & Smoker & Male & White & 54 & 0.11 & 2.10 & 7136 & \\
\hline 30 & Smoker & Male & White & & 0.18 & 4.87 & $N A^{b}$ & \\
\hline 31 & Smoker & Male & White & 60 & 0.13 & 1.82 & 1324 & 0.99 \\
\hline 32 & Smoker & Female & White & 58 & 0.16 & 2.92 & 1367 & 1.95 \\
\hline 33 & Smoker & Female & White & 63 & 0.10 & 2.12 & 6 & 1.03 \\
\hline 34 & Smoker & Female & White & 70 & 0.14 & 3.88 & 2386 & 0.21 \\
\hline 35 & Smoker & Female & White & 59 & 0.13 & 2.24 & 1092 & 0.22 \\
\hline 36 & Smoker & Female & White & 67 & 0.12 & 5.99 & 591 & 0.31 \\
\hline 37 & Smoker & Male & White & 63 & 0.13 & 2.24 & 2352 & 0.73 \\
\hline \multicolumn{3}{|c|}{ Smoker average $\pm S D^{a}$} & & $61 \pm 8$ & $0.13 \pm 0.03$ & $2.88 \pm 1.39$ & $\begin{array}{c}2131 \pm \\
2030\end{array}$ & $1.11 \pm 1.10$ \\
\hline \multicolumn{3}{|c|}{ Total average $\pm \mathrm{SD}^{\mathrm{a}}$} & & $63 \pm 9$ & $0.13 \pm 0.04$ & $2.77 \pm 1.33$ & & \\
\hline
\end{tabular}

a. SD: standard deviation

b. NA: not available 
Table S2. Levels of AP sites (PMOA-dR) in human leukocyte DNA and subject information

\begin{tabular}{|c|c|c|c|c|c|c|c|c|}
\hline Subject & Study & $\begin{array}{c}\text { Smoking } \\
\text { status }\end{array}$ & Gender & Race & Age & $\mathrm{dG}(\mu \mathrm{mol})$ & $\begin{array}{c}\text { PMOA-dR } \\
\left.\text { (per } 10^{7} \mathrm{nts}\right)\end{array}$ & $\begin{array}{c}\text { NNAL } \\
\text { (pmol/mL) }\end{array}$ \\
\hline 1 & 3-Arm & nonsmoker & Female & Black & 20 & 0.026 & 1.81 & \\
\hline 2 & 3-Arm & nonsmoker & Female & Black & 20 & 0.023 & 1.56 & \\
\hline 3 & UMN & nonsmoker & Male & White & 20 & 0.07 & 1.49 & \\
\hline 4 & UMN & nonsmoker & Male & White & 21 & 0.05 & 1.9 & \\
\hline 5 & 3-Arm & nonsmoker & Female & Asian & 23 & 0.062 & 2.50 & \\
\hline 6 & 3-Arm & nonsmoker & Male & Asian & 24 & 0.001 & 0.70 & \\
\hline 7 & 3-Arm & nonsmoker & Female & White & 25 & 0.063 & 2.12 & \\
\hline 8 & 3-Arm & nonsmoker & Female & White & 25 & 0.063 & 1.88 & \\
\hline 9 & 3-Arm & nonsmoker & Female & White & 25 & 0.047 & 2.56 & \\
\hline 10 & 3-Arm & nonsmoker & Female & White & 27 & 0.077 & 2.14 & \\
\hline 11 & 3-Arm & nonsmoker & Female & White & 28 & 0.041 & 1.60 & \\
\hline 12 & 3-Arm & nonsmoker & Female & White & 28 & 0.055 & 2.58 & \\
\hline 13 & 3-Arm & nonsmoker & Male & Black & 29 & 0.036 & 2.09 & \\
\hline 14 & 3-Arm & nonsmoker & Female & Black & 31 & 0.034 & 1.26 & \\
\hline 15 & UMN & nonsmoker & Female & White & 34 & 0.07 & 2.26 & \\
\hline 16 & 3-Arm & nonsmoker & Female & Asian & 35 & 0.030 & 2.55 & \\
\hline 17 & 3-Arm & nonsmoker & Female & White & 35 & 0.062 & 3.47 & \\
\hline 18 & 3-Arm & nonsmoker & Female & White & 37 & 0.095 & 2.03 & \\
\hline 19 & 3-Arm & nonsmoker & Female & Black & 39 & 0.034 & 2.11 & \\
\hline 20 & 3-Arm & nonsmoker & Female & White & 41 & 0.020 & 1.63 & \\
\hline 21 & 3-Arm & nonsmoker & Female & Asian & 44 & 0.076 & 2.40 & \\
\hline 22 & 3-Arm & nonsmoker & Male & White & 44 & 0.006 & 1.22 & \\
\hline 23 & 3-Arm & nonsmoker & Female & Black & 45 & 0.07 & 2.70 & \\
\hline 24 & 3-Arm & nonsmoker & Female & Black & 45 & 0.041 & 1.83 & \\
\hline 25 & 3-Arm & nonsmoker & Male & White & 45 & 0.054 & 2.39 & \\
\hline 26 & 3-Arm & nonsmoker & Female & Black & 48 & 0.055 & 1.86 & \\
\hline 27 & UMN & nonsmoker & Female & White & 48 & 0.12 & 2.02 & \\
\hline 28 & 3-Arm & nonsmoker & Female & Black & 49 & 0.035 & 2.08 & \\
\hline 29 & UMN & nonsmoker & Female & White & 50 & 0.1 & 2.61 & \\
\hline 30 & 3-Arm & nonsmoker & Female & White & 54 & 0.042 & 2.28 & \\
\hline 31 & 3-Arm & nonsmoker & Male & Black & 61 & 0.034 & 3.50 & \\
\hline \multicolumn{3}{|c|}{ Nonsmoker average $\pm \mathrm{SD}^{\mathrm{a}}$} & & & $35 \pm 11$ & $0.05 \pm 0.03$ & $2.10 \pm 0.59$ & \\
\hline 32 & UMN & smoker & Male & White & 21 & 0.07 & 2.42 & \\
\hline 33 & 3-Arm & smoker & Male & Black & 24 & 0.035 & 2.06 & 1.60 \\
\hline 34 & 3-Arm & smoker & Female & Black & 27 & 0.055 & 1.83 & 1.33 \\
\hline 35 & 3-Arm & smoker & Female & A-Indian* & 30 & 0.053 & 1.47 & 0.59 \\
\hline 36 & 3-Arm & smoker & Male & White & 30 & 0.026 & 1.70 & 0.33 \\
\hline 37 & 3-Arm & smoker & Female & Black & 38 & 0.083 & 1.76 & 1.01 \\
\hline 38 & UMN & smoker & Female & White & 38 & 0.11 & 2.54 & \\
\hline 39 & 3-Arm & smoker & Male & Black & 39 & 0.033 & 1.82 & 0.50 \\
\hline 40 & 3-Arm & smoker & Female & Black & 39 & 0.072 & 1.90 & 0.37 \\
\hline 41 & UMN & smoker & Female & White & 41 & 0.11 & 2.33 & \\
\hline 42 & 3-Arm & smoker & Male & Black & 43 & 0.038 & 2.36 & 0.76 \\
\hline 43 & 3-Arm & smoker & Male & White & 43 & 0.039 & 1.35 & 0.63 \\
\hline 44 & 3-Arm & smoker & Female & Black & 45 & 0.011 & 1.77 & 0.17 \\
\hline 45 & 3-Arm & smoker & Male & White & 45 & 0.064 & 2.28 & 0.50 \\
\hline 46 & 3-Arm & smoker & Male & A-Indian* & 46 & 0.073 & 2.43 & 1.53 \\
\hline 47 & 3-Arm & smoker & Male & Black & 46 & 0.035 & 2.16 & 0.59 \\
\hline 48 & 3-Arm & smoker & Male & Black & 47 & 0.033 & 1.38 & 0.17 \\
\hline 49 & 3-Arm & smoker & Female & Black & 47 & 0.024 & 1.59 & 0.80 \\
\hline 50 & 3-Arm & smoker & Female & White & 47 & 0.084 & 2.52 & 1.76 \\
\hline 51 & 3-Arm & smoker & Male & Black & 49 & 0.057 & 2.30 & 1.77 \\
\hline 52 & UMN & smoker & Male & White & 49 & 0.05 & 2.11 & \\
\hline 53 & 3-Arm & smoker & Male & A-Indian* & 50 & 0.024 & 1.96 & 0.64 \\
\hline 54 & 3-Arm & smoker & Female & White & 50 & 0.034 & 2.49 & 0.375 \\
\hline
\end{tabular}




\begin{tabular}{|c|c|c|c|c|c|c|c|c|}
\hline 55 & 3-Arm & smoker & Female & Black & 51 & 0.057 & 2.33 & 1.51 \\
\hline 56 & 3-Arm & smoker & Female & Black & 52 & 0.024 & 1.16 & 0.44 \\
\hline 57 & 3-Arm & smoker & Male & White & 52 & 0.071 & 2.21 & 2.04 \\
\hline 58 & 3-Arm & smoker & Female & Black & 55 & 0.059 & 4.57 & 2.29 \\
\hline 59 & 3-Arm & smoker & Male & Black & 58 & 0.044 & 2.01 & 0.31 \\
\hline 60 & 3-Arm & smoker & Male & Black & 58 & 0.059 & 1.8 & 2.47 \\
\hline 61 & 3-Arm & smoker & Male & White & 63 & 0.048 & 1.67 & 0.675 \\
\hline 62 & UMN & smoker & Female & White & 63 & 0.21 & 1.86 & \\
\hline \multicolumn{3}{|c|}{ Smoker average $\pm \mathrm{SD}^{\mathrm{a}}$} & & & $45 \pm 10$ & $0.06 \pm 0.04$ & $2.07 \pm 0.60$ & $0.97 \pm 0.69$ \\
\hline \multicolumn{3}{|c|}{ Total average $\pm \mathrm{SD}^{\mathrm{a}}$} & & & $40 \pm 12$ & $0.05 \pm 0.03$ & $2.08 \pm 0.59$ & \\
\hline
\end{tabular}

${ }^{*}$ A-Indian: American Indian

a. SD: standard deviation

Table S3. AP site levels (PMOA-dR, average \pm standard deviation) in control rat lung analyzed along with human samples.

\begin{tabular}{|c|c|c|c|c|}
\hline Experimental approach & Test \# & $\mathrm{dG}(\mu \mathrm{mol})$ & $\begin{array}{c}\text { PMOA-dR } \\
\left(\text { per } 10^{7} \mathrm{nts}\right)\end{array}$ & $\begin{array}{c}\% \text { change } \\
\text { of AP site }\end{array}$ \\
\hline Figure S1 procedure & Test 1 & $0.21 \pm 0.01$ & $1.68 \pm 0.27$ & \\
\hline Figure S1 procedure & Test 2 & $0.17 \pm 0.01$ & $1.49 \pm 0.15$ & \\
\hline Figure S1 procedure & Test 3 & $0.18 \pm 0.02$ & $1.43 \pm 0.09$ & \\
\hline Figure S1 procedure & Test 4 & $0.18 \pm 0.01$ & $1.43 \pm 0.12$ & \\
\hline Figure S1 procedure & Test 5 & $0.19 \pm 0.03$ & $1.66 \pm 0.12$ & \\
\hline Figure S1 procedure & Test 6 & $0.19 \pm 0.03$ & $1.95 \pm 0.19$ & \\
\hline PMOA added with SDS and incubate $3 \mathrm{~h}^{\mathrm{a}}$ & Test 7 & $0.22 \pm 0.01$ & $2.06 \pm 0.05$ & $24 \%^{\mathrm{b}}$ \\
\hline PMOA added with SDS and incubate $1.5 \mathrm{~h}^{\mathrm{c}}$ & Test 8 & $0.16 \pm 0.01$ & $1.19 \pm 0.08$ & $-39 \%^{\mathrm{d}}$ \\
\hline
\end{tabular}

a. $\quad$ Test 7 was conducted at the same time with Test 6 from the same rat tissue;

b. Relative to Test 6;

c. Test 8 was conducted at the same time with Test 5 from the same rat tissue;

d. Relative to Test 5. 
Table S4. dG levels (average \pm standard deviation) in rat tissues

\begin{tabular}{|c|c|c|c|c|c|}
\hline \multirow{2}{*}{\multicolumn{2}{|c|}{ Sample type }} & $\mathrm{dG}$ & \multirow{2}{*}{\multicolumn{2}{|c|}{ Sample type }} & $d G$ \\
\hline & & $\mu \mathrm{mol}$ & & & $\mu \mathrm{mol}$ \\
\hline \multicolumn{3}{|c|}{ NNK acute exposure } & \multicolumn{3}{|c|}{ 14ppm (S)-NNN for 10 weeks } \\
\hline \multirow{3}{*}{ liver } & control & $0.094 \pm 0.010$ & \multirow{2}{*}{$\begin{array}{c}\text { esophageal } \\
\text { mucosa }\end{array}$} & control & $0.059 \pm 0.007$ \\
\hline & low $(25$ umol $/ \mathrm{kg})$ & $0.094 \pm 0.032$ & & exposure & $0.057 \pm 0.004$ \\
\hline & high (100umol/kg) & $0.093 \pm 0.021$ & \multirow{2}{*}{$\begin{array}{c}\text { oral } \\
\text { mucosa }\end{array}$} & control & $0.139 \pm 0.047$ \\
\hline \multirow{3}{*}{ lung } & control & $0.119 \pm 0.027$ & & exposure & $0.107 \pm 0.038$ \\
\hline & low (25 umol/kg) & $0.197 \pm 0.072$ & \multirow{2}{*}{$\begin{array}{c}\text { nasal } \\
\text { respiratory } \\
\text { mucosa }\end{array}$} & control & $0.074 \pm 0.029$ \\
\hline & high (100umol/kg) & $0.103 \pm 0.017$ & & exposure & $0.074 \pm 0.018$ \\
\hline \multicolumn{3}{|c|}{ 5ppm NNK chronic exposure } & \multirow{2}{*}{$\begin{array}{c}\text { nasal } \\
\text { olfactory } \\
\text { mucosa }\end{array}$} & control & $0.172 \pm 0.031$ \\
\hline \multirow{8}{*}{ liver } & 10 week control & $0.105 \pm 0.016$ & & exposure & $0.167 \pm 0.023$ \\
\hline & 10 week exposure & $0.109 \pm 0.012$ & \multirow{2}{*}{ liver } & control & $0.105 \pm 0.009$ \\
\hline & 30 week control & $0.106 \pm 0.009$ & & exposure & $0.107 \pm 0.010$ \\
\hline & 30 week exposure & $0.092 \pm 0.014$ & \multirow{2}{*}{ lung } & control & $0.279 \pm 0.075$ \\
\hline & 50 week control & $0.114 \pm 0.022$ & & exposure & $0.331 \pm 0.020$ \\
\hline & 50 week exposure & $0.111 \pm 0.008$ & & & \\
\hline & 70 week control & $0.141 \pm 0.021$ & & & \\
\hline & 70 week exposure & $0.130 \pm 0.058$ & & & \\
\hline \multicolumn{6}{|c|}{ NNKOAc in vitro exposure } \\
\hline \multirow{5}{*}{ liver } & control & $0.090 \pm 0.001$ & & & \\
\hline & $2 \mu \mathrm{M}$ & $0.083 \pm 0.003$ & & & \\
\hline & $12 \mu \mathrm{M}$ & $0.088 \pm 0.002$ & & & \\
\hline & $49 \mu \mathrm{M}$ & $0.086 \pm 0.010$ & & & \\
\hline & $243 \mu \mathrm{M}$ & $0.089 \pm 0.004$ & & & \\
\hline \multirow{5}{*}{ lung } & control & $0.107 \pm 0.012$ & & & \\
\hline & $2 \mu \mathrm{M}$ & $0.125 \pm 0.016$ & & & \\
\hline & $12 \mu \mathrm{M}$ & $0.107 \pm 0.014$ & & & \\
\hline & $49 \mu \mathrm{M}$ & $0.141 \pm 0.006$ & & & \\
\hline & $243 \mu \mathrm{M}$ & $0.134 \pm 0.013$ & & & \\
\hline
\end{tabular}


Table S5a. Comparison of POB-DNA adduct levels (average \pm standard deviation) from NNK acute exposures (unit: per $10^{7} \mathrm{nts}$ )

\begin{tabular}{|c|c|c|c|c|c|}
\hline \multirow[b]{2}{*}{ Tissue } & \multirow{2}{*}{$\begin{array}{l}\text { NNK Dose } \\
\text { (mg/kg bw) }\end{array}$} & \multicolumn{2}{|c|}{$\mathrm{N}^{7} \mathrm{POBG}$} & \multicolumn{2}{|c|}{$\mathrm{O}^{2} \mathrm{POBdT}$} \\
\hline & & $\begin{array}{c}\text { Wang } 2007 \\
\text { (1) }\end{array}$ & This study & Wang 2007 (1) & This study \\
\hline \multirow{2}{*}{ lung } & 5.2 & $3.11 \pm 0.30$ & $1.23 \pm 0.08$ & $3.73 \pm 0.22$ & $4.84 \pm 1.96$ \\
\hline & 20.7 & $6.00 \pm 1.59$ & $1.68 \pm 0.11$ & $6.73 \pm 1.61$ & $7.68 \pm 1.95$ \\
\hline \multirow{2}{*}{ liver } & 5.2 & $11.83 \pm 5.33$ & $4.29 \pm 0.46$ & $11.77 \pm 2.42$ & $21.78 \pm 5.94$ \\
\hline & 20.7 & $40.67 \pm 5.33$ & $13.56 \pm 1.25$ & $41.00 \pm 5.63$ & $39.64 \pm 14.58$ \\
\hline
\end{tabular}

Table S5b. Comparison of POB-DNA adduct levels (average \pm standard deviation) from NNK chronic exposures (unit: per $10^{7} \mathrm{nts}$ ).

\begin{tabular}{|c|c|c|c|c|c|}
\hline Tissue & Dose & Time (week) & N7POBG & $\mathrm{O}^{2} \mathrm{POBdT}$ & Ref. \\
\hline \multirow{4}{*}{ Lung } & \multirow{4}{*}{$\begin{array}{c}5 \text { ppm NNK } \\
\text { in drinking } \\
\text { water }\end{array}$} & 10 & $3.23 \pm 0.49$ & $11.97 \pm 1.38$ & \multirow{4}{*}{ Balbo 2014 (2) } \\
\hline & & 30 & $2.50 \pm 0.10$ & $16.03 \pm 0.64$ & \\
\hline & & 50 & $2.29 \pm 0.22$ & $14.70 \pm 1.07$ & \\
\hline & & 70 & $1.05 \pm 0.25$ & $6.61 \pm 1.56$ & \\
\hline \multirow{6}{*}{ Liver } & \multirow{6}{*}{$\begin{array}{c}10 \mathrm{ppm} \\
\text { NNK in } \\
\text { drinking } \\
\text { water }\end{array}$} & 1 & $1.63 \pm 0.35$ & $2.17 \pm 0.40$ & \multirow{6}{*}{ Lao 2007 (3) } \\
\hline & & 2 & $2.93 \pm 0.61$ & $4.10 \pm 0.91$ & \\
\hline & & 5 & $3.50 \pm 0.30$ & $7.30 \pm 0.58$ & \\
\hline & & 10 & $4.87 \pm 2.08$ & $12.47 \pm 3.9$ & \\
\hline & & 16 & $3.90 \pm 0.29$ & $11.80 \pm 2.14$ & \\
\hline & & 20 & $2.43 \pm 0.75$ & $8.93 \pm 2.14$ & \\
\hline \multirow{4}{*}{ Liver } & \multirow{4}{*}{$\begin{array}{c}5 \text { ppm NNK } \\
\text { in drinking } \\
\text { water }\end{array}$} & 10 & $0.74 \pm 0.18$ & $3.17 \pm 0.74$ & \multirow{4}{*}{ This study } \\
\hline & & 30 & $0.51 \pm 0.11$ & $3.75 \pm 0.79$ & \\
\hline & & 50 & $0.42 \pm 0.07$ & $3.26 \pm 0.30$ & \\
\hline & & 70 & $0.24 \pm 0.06$ & $1.95 \pm 0.65$ & \\
\hline
\end{tabular}

Table S6. Comparison of POB-DNA adduct levels (average \pm standard deviation) from (S)-NNN exposure (unit: per $10^{7} \mathrm{nts}$ )

\begin{tabular}{|c|c|c|c|c|c|c|}
\hline & $\begin{array}{c}\text { Yang } 2016 \\
\text { (4) }\end{array}$ & $\begin{array}{c}\text { Zhao } 2013 \\
\text { (5) }\end{array}$ & This study & $\begin{array}{c}\text { Yang } 2016 \\
\text { (4) }\end{array}$ & $\begin{array}{c}\text { Zhao } 2013 \\
(5)\end{array}$ & This study \\
\hline $\begin{array}{c}\text { Dose and treated } \\
\text { time (in drinking } \\
\text { water) }\end{array}$ & $\begin{array}{l}14 \mathrm{ppm} \\
\text { NNN for } 5 \\
\text { weeks }\end{array}$ & $\begin{array}{l}14 \mathrm{ppm} \\
\text { NNN for } 10 \\
\text { weeks }\end{array}$ & & $\begin{array}{l}14 \mathrm{ppm} \\
\text { NNN for } 5 \\
\text { weeks }\end{array}$ & $\begin{array}{l}14 \mathrm{ppm} \\
\text { NNN for } 10 \\
\text { weeks }\end{array}$ & \\
\hline Tissue Chemical & $\mathrm{N}^{7} \mathrm{POBG}$ & N7POBG & $\mathrm{N}^{7} \mathrm{PC}$ & $\mathrm{O}^{2} \mathrm{POBdT}$ & $\mathrm{O}^{2} \mathrm{POBdT}$ & $\mathrm{O}^{2} \mathrm{POBdT}$ \\
\hline Oral mucosa & $0.54 \pm 0.13$ & $0.90 \pm 0.09$ & 283 & $0.67 \pm 0.16$ & $1.07 \pm 0.16$ & 087 \\
\hline $\begin{array}{r}\text { Esol } \\
\text { mi }\end{array}$ & $1.62 \pm 0.24$ & $4.23 \pm 1.03$ & $2.43 \pm 0.52$ & $1.58 \pm 0.27$ & $2.52 \pm 0.42$ & $2.54 \pm 0.39$ \\
\hline $\begin{array}{r}\text { Nasal re } \\
\text { mu }\end{array}$ & $2.26 \pm 0.62$ & $4.34 \pm 0.08$ & $3.05 \pm 0.63$ & $5.70 \pm 1.10$ & $18.06 \pm 1.19$ & $8.36 \pm 1.02$ \\
\hline $\begin{array}{c}\text { Nasal olfactory } \\
\text { mucosa }\end{array}$ & $0.32 \pm 0.04$ & $0.33 \pm 0.01$ & $0.48 \pm 0.11$ & $0.88 \pm 0.01$ & $1.10 \pm 0$ & $1.78 \pm 0.28$ \\
\hline & $.19 \pm 0.02$ & $0.75 \pm 0.07$ & & $0.80 \pm 0.12$ & $1.68 \pm 0.27$ & $1.06 \pm 0.20$ \\
\hline Lung & $0.28 \pm 0.01$ & $0.32 \pm 0.01$ & $0.07 \pm 0.03$ & $0.69 \pm 0.05$ & $0.77 \pm 0.02$ & $0.33 \pm 0.08$ \\
\hline
\end{tabular}


Table S7. Comparison of DNA adducts and AP sites (PMOA-dR) in cigarette smokers and nonsmokers (average \pm standard deviation, unit: per $10^{7} \mathrm{nts}$ )

\begin{tabular}{|c|c|c|c|c|c|c|c|}
\hline \multirow{2}{*}{ DNA adduct } & \multirow{2}{*}{ Possible sources } & \multirow{2}{*}{ Tissue } & \multicolumn{2}{|r|}{ Smoker } & \multicolumn{2}{|c|}{ Nonsmoker } & \multirow[t]{2}{*}{ Ref. } \\
\hline & & & $\mathrm{N}$ & Mean \pm SD & $\mathrm{N}$ & Mean \pm SD & \\
\hline Acr-dG ${ }^{\#}$ & $\begin{array}{l}\text { acrolein from } \\
\text { incomplete } \\
\text { combustion, } \\
\text { cigarette smoke or } \\
\text { endogenous lipid } \\
\text { peroxidation }\end{array}$ & Lung & 19 & $0.29 \pm 0.15$ & 18 & $0.25 \pm 0.11$ & $\begin{array}{c}\text { Yang } 2019 \\
\text { (6) }\end{array}$ \\
\hline $\begin{array}{l}\text { Total methyl } \\
\text { phosphates }\end{array}$ & $\begin{array}{l}\text { methylating } \\
\text { carcinogens }\end{array}$ & Lung & 13 & $\begin{array}{l}0.13 \pm \\
0.06^{*}\end{array}$ & 17 & $0.08 \pm 0.04$ & $\begin{array}{l}\text { Ma } 2019 \\
(7)\end{array}$ \\
\hline $\begin{array}{l}\text { BPDE- } N^{2}- \\
\quad \mathrm{dG}\end{array}$ & $\begin{array}{l}\text { benzo[a]pyrene from } \\
\text { human diet, } \\
\text { polluted air, } \\
\text { cigarette smoke, or } \\
\text { incomplete } \\
\text { combustion } \\
\end{array}$ & Lung & 11 & $\begin{array}{l}0.00031 \pm \\
0.00022^{*}\end{array}$ & 18 & $\begin{array}{c}0.00013 \pm \\
0.00008\end{array}$ & $\begin{array}{c}\text { Villalta } \\
2017(8)\end{array}$ \\
\hline PMOA-dR & $\begin{array}{c}\text { NNK or NNN } \\
\text { exposure }\end{array}$ & Lung & 20 & $2.88 \pm 1.39$ & 17 & $2.65 \pm 1.29$ & This study \\
\hline Acr-dG & $\begin{array}{l}\text { acrolein from } \\
\text { incomplete } \\
\text { combustion, } \\
\text { cigarette smoke or } \\
\text { endogenous lipid } \\
\text { peroxidation } \\
\end{array}$ & Leukocyte & 25 & $\begin{array}{c}0.074 \pm \\
0.034\end{array}$ & 25 & $\begin{array}{l}0.098 \pm \\
0.055\end{array}$ & $\begin{array}{l}\text { Zhang } \\
2011(9)\end{array}$ \\
\hline $\mathrm{N}^{2}-\mathrm{CEG}$ & $\begin{array}{c}\text { exposure to } \\
\text { mutagenic } \\
\text { methylglyoxal from } \\
\text { food or cigarette } \\
\text { smoke }\end{array}$ & Leukocyte & 20 & $9.89 \pm 5.69$ & 20 & $10.23 \pm 6.27$ & $\begin{array}{c}\text { Cheng } \\
2020(10)\end{array}$ \\
\hline 7-CEG & $\begin{array}{l}\text { metabolism of the } \\
\text { carcinogenic } \\
\text { nitrosamines 3- } \\
\text { (methylnitrosamino) } \\
\text { propionic acid and } \\
\text { 1-nitroso-5,6- } \\
\text { dihydrouracil, or by } \\
\text { reaction of DNA with } \\
\text { acrylic acid } \\
\end{array}$ & Leukocyte & 20 & $1.29 \pm 0.52$ & 20 & $1.19 \pm 0.47$ & $\begin{array}{l}\text { Cheng } \\
2010 \text { and } \\
2020 \\
(10,11)\end{array}$ \\
\hline $\begin{array}{l}\mathrm{N}^{6}-\mathrm{HOMe}- \\
\text { dAdo }\end{array}$ & $\begin{array}{c}\text { formaldehyde } \\
\text { exposure or NNK or } \\
\text { nicotine metabolism }\end{array}$ & Leukocyte & 32 & $\begin{array}{l}0.51 \pm \\
0.59^{*}\end{array}$ & 30 & $0.04 \pm 0.10$ & $\begin{array}{c}\text { Wang } \\
2009(12)\end{array}$ \\
\hline 7-ethyl-G & $\begin{array}{l}\text { ethanol metabolism } \\
\text { or degradation } \\
\text { of DNA adducts with } \\
\text { more complex } \\
\text { structures }\end{array}$ & Leukocyte & 30 & $\begin{array}{l}0.106 \pm \\
0.093\end{array}$ & 30 & $\begin{array}{l}0.088 \pm \\
0.075\end{array}$ & $\begin{array}{c}\text { Balbo } \\
2011(13)\end{array}$ \\
\hline PMOA-dR & $\begin{array}{c}\text { NNK or NNN } \\
\text { exposure }\end{array}$ & Leukocyte & 31 & $2.07 \pm 0.60$ & 31 & $2.10 \pm 0.59$ & This study \\
\hline
\end{tabular}

"Sum of $\alpha-A c r-d G$ and $\gamma-A c r-d G$.

*Smokers are significantly higher than nonsmokers. 
Table S8. AP site levels (average \pm standard deviation) in human leukocyte DNA based on subject smoking status, gender, race and age (unit: PMOA-dR per $10^{7} \mathrm{nts}$ )

\begin{tabular}{|c|c|c|c|c|c|c|c|c|}
\hline \multicolumn{2}{|c|}{ Category } & \multicolumn{2}{|c|}{ Nonsmokers } & \multicolumn{2}{|c|}{ Smokers } & \multirow{2}{*}{$\begin{array}{c}p \text { value } \\
\text { ( nonsmokers } \\
\text { vs. smokers) }\end{array}$} & \multicolumn{2}{|r|}{ Total } \\
\hline & & $\mathrm{N}$ & Mean \pm SD & $\mathrm{N}$ & Mean \pm SD & & $\mathrm{N}$ & Mean \pm SD \\
\hline \multirow{3}{*}{ Gender } & Female $(\mathrm{F})$ & 24 & $2.16 \pm 0.47$ & 14 & $2.15 \pm 0.81$ & 0.482 & 38 & $2.16 \pm 0.61$ \\
\hline & Male (M) & 7 & $1.90 \pm 0.90$ & 17 & $2.00 \pm 0.34$ & 0.343 & 24 & $1.97 \pm 0.54$ \\
\hline & $\begin{array}{c}\mathrm{p} \text { value } \mathrm{F} \text { vs. } \\
M\end{array}$ & \multicolumn{2}{|r|}{0.155} & \multicolumn{2}{|r|}{0.247} & & \multicolumn{2}{|r|}{0.114} \\
\hline \multirow{4}{*}{ Race } & $\begin{array}{c}\text { Asian/Indian } \\
\text { American }\end{array}$ & $4^{a}$ & $2.04 \pm 0.90^{a}$ & $3^{b}$ & $1.96 \pm 0.48^{b}$ & & 7 & $2.00 \pm 0.69$ \\
\hline & Black (B) & 10 & $2.08 \pm 0.63$ & 16 & $2.05 \pm 0.75$ & 0.458 & 26 & $2.06 \pm 0.72$ \\
\hline & White (W) & 17 & $2.13 \pm 0.53$ & 12 & $2.12 \pm 0.39$ & 0.488 & 29 & $2.13 \pm 0.47$ \\
\hline & $\begin{array}{c}p \text { value } B \text { vs. } \\
\text { W }\end{array}$ & & 0.414 & \multicolumn{2}{|r|}{0.378} & & \multicolumn{2}{|r|}{0.339} \\
\hline \multirow{3}{*}{ Age } & 20-31 $(\mathrm{Y})$ & 14 & $1.87 \pm 0.53$ & 5 & $1.90 \pm 0.36$ & 0.463 & 19 & $1.88 \pm 0.48$ \\
\hline & $31+(\mathrm{O})$ & 17 & $2.29 \pm 0.58$ & 26 & $2.10 \pm 0.63$ & 0.164 & 43 & $2.18 \pm 0.61$ \\
\hline & $\begin{array}{c}p \text { value } \mathrm{Y} \text { vs. } \\
\mathrm{O}\end{array}$ & & $0.023^{*}$ & \multicolumn{2}{|r|}{0.244} & & \multirow{2}{*}{\multicolumn{2}{|c|}{$0.032^{*}$}} \\
\hline Total & & 31 & $2.10 \pm 0.59$ & 31 & $2.07 \pm 0.6$ & 0.414 & & \\
\hline
\end{tabular}

a. Asian American; b. Indian American

*31+ group is significantly higher than $20-31$ group. 


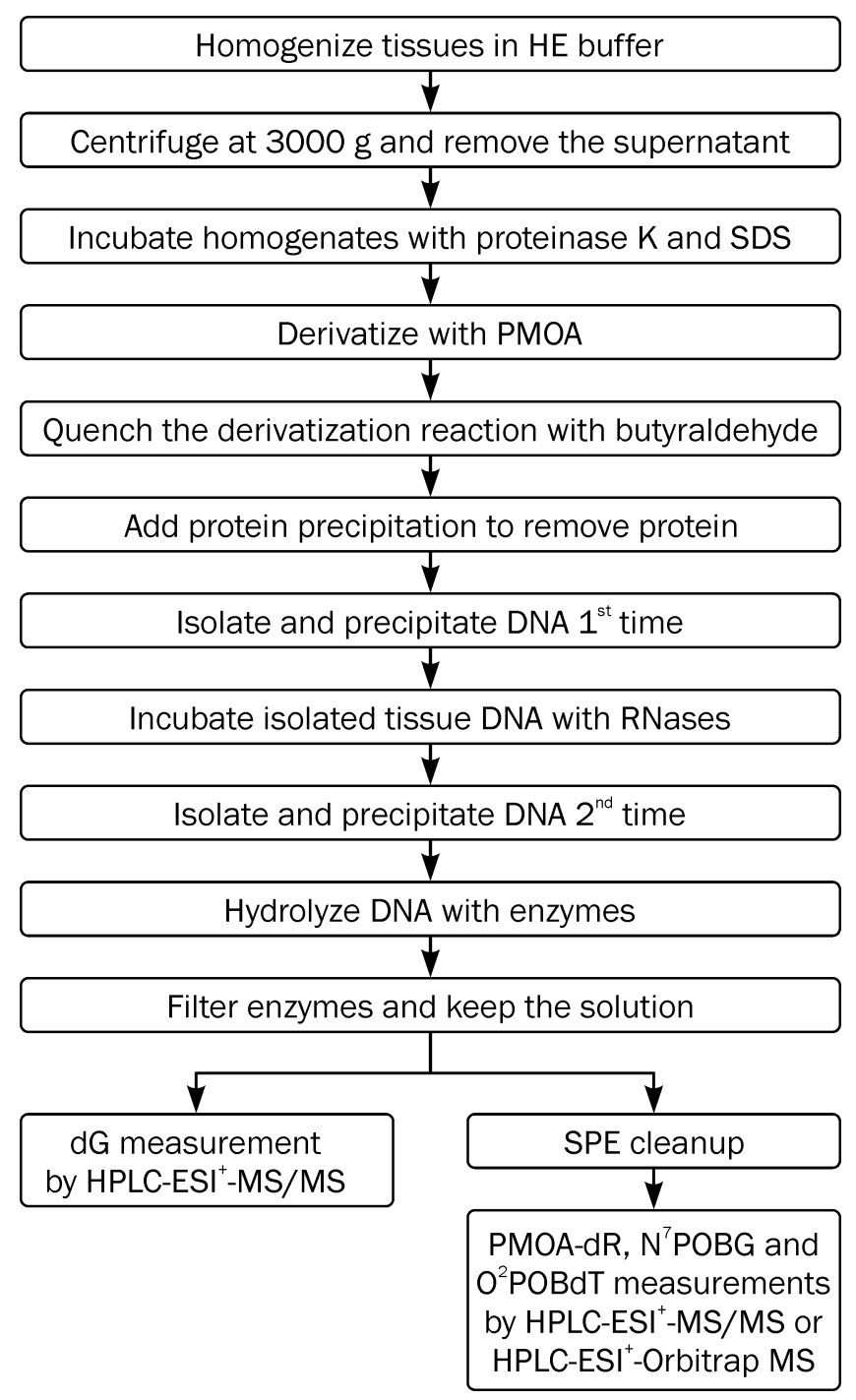

Figure S1. Flow chart of the experimental procedure for analysis of AP sites and DNA adducts 

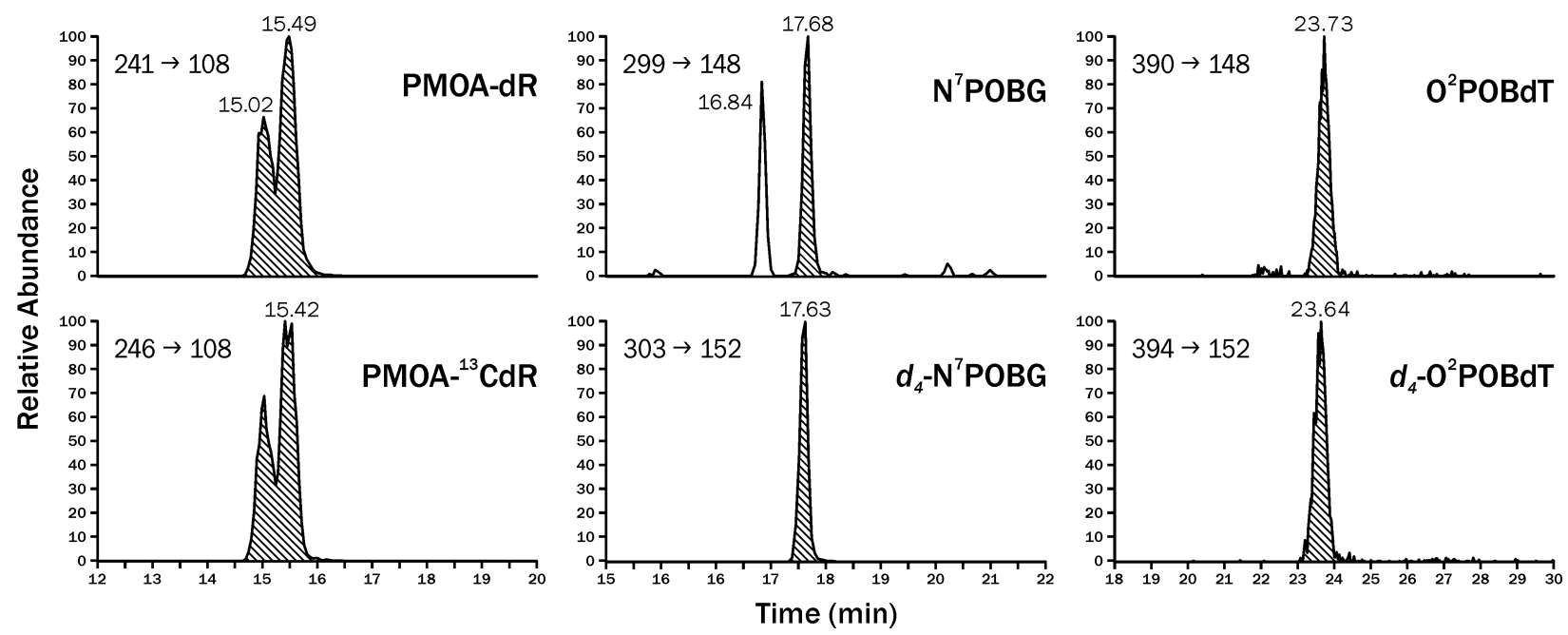

Figure S2. HPLC-ESI-MS/MS chromatograms of PMOA-dR and POB-DNA adducts in nasal respiratory mucosa DNA of rats treated for 10 weeks with (S)-NNN. PMOA-dR (and PMOA${ }^{13} \mathrm{CdR}$ ) and $\mathrm{N}^{7} \mathrm{POBG}$ (and $d_{4}-\mathrm{N}^{7} \mathrm{POBG}$ ) were analyzed with a high resolution LTQ Orbitrap Velos mass spectrometer, and $\mathrm{O}^{2} \mathrm{POBdT}$ (and $d_{4}-\mathrm{O}^{2} \mathrm{POBdT}$ ) was analyzed with a TSQ quantum Vantage triple quadrupole mass spectrometer. 

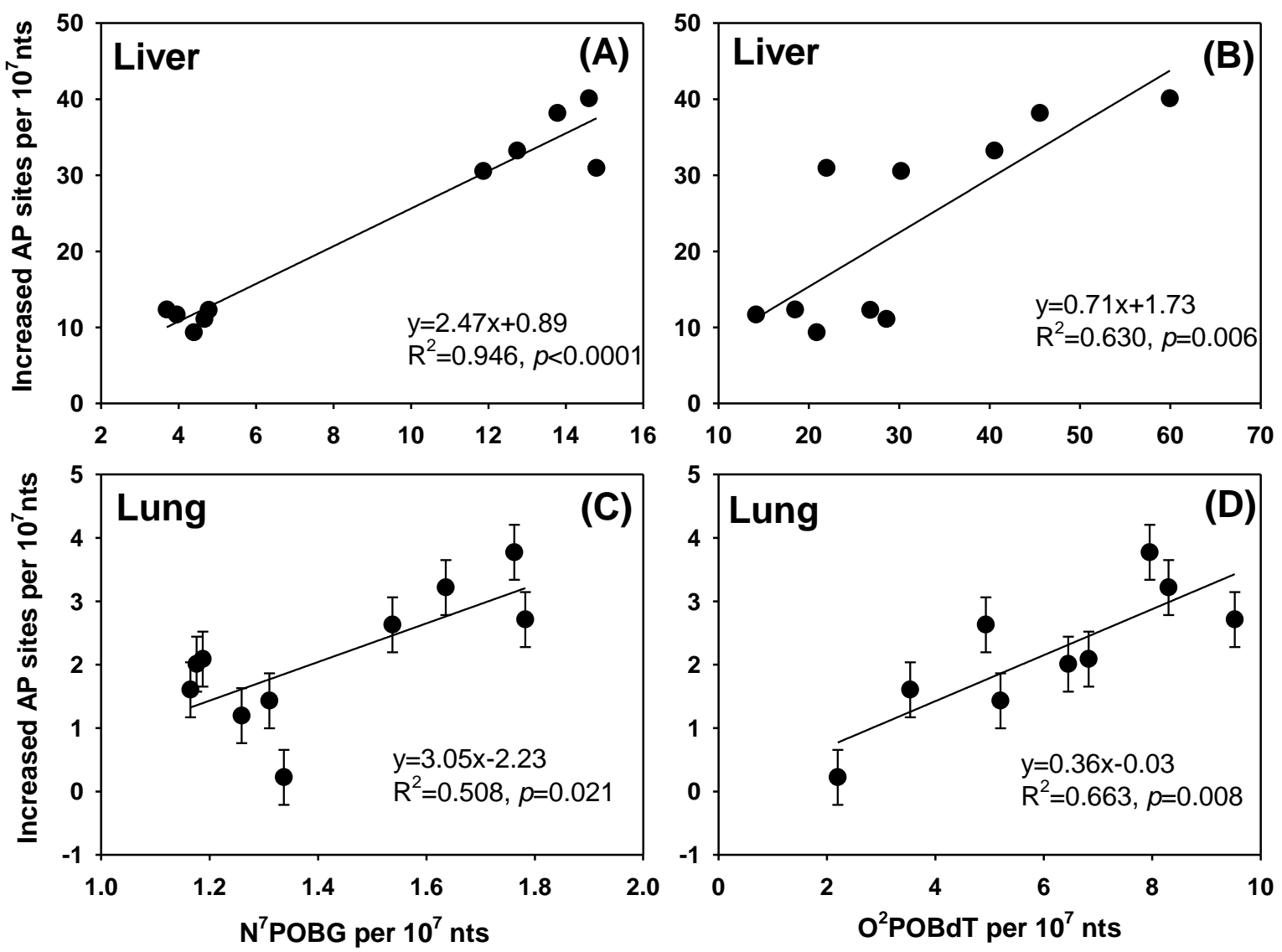

Figure S3. Dependence of increased AP site formation (exposures minus average controls) on $\mathrm{N}^{7} \mathrm{POBG}(\mathrm{A}, \mathrm{C})$ or $\mathrm{O}^{2} \mathrm{POBdT}(\mathrm{B}, \mathrm{D})$ in lung and liver of rats treated with $5.2 \mathrm{mg} / \mathrm{kg}$ and 20.7 $\mathrm{mg} / \mathrm{kg}$ NNK for 4 days. The error bars in liver are too small to be seen. 

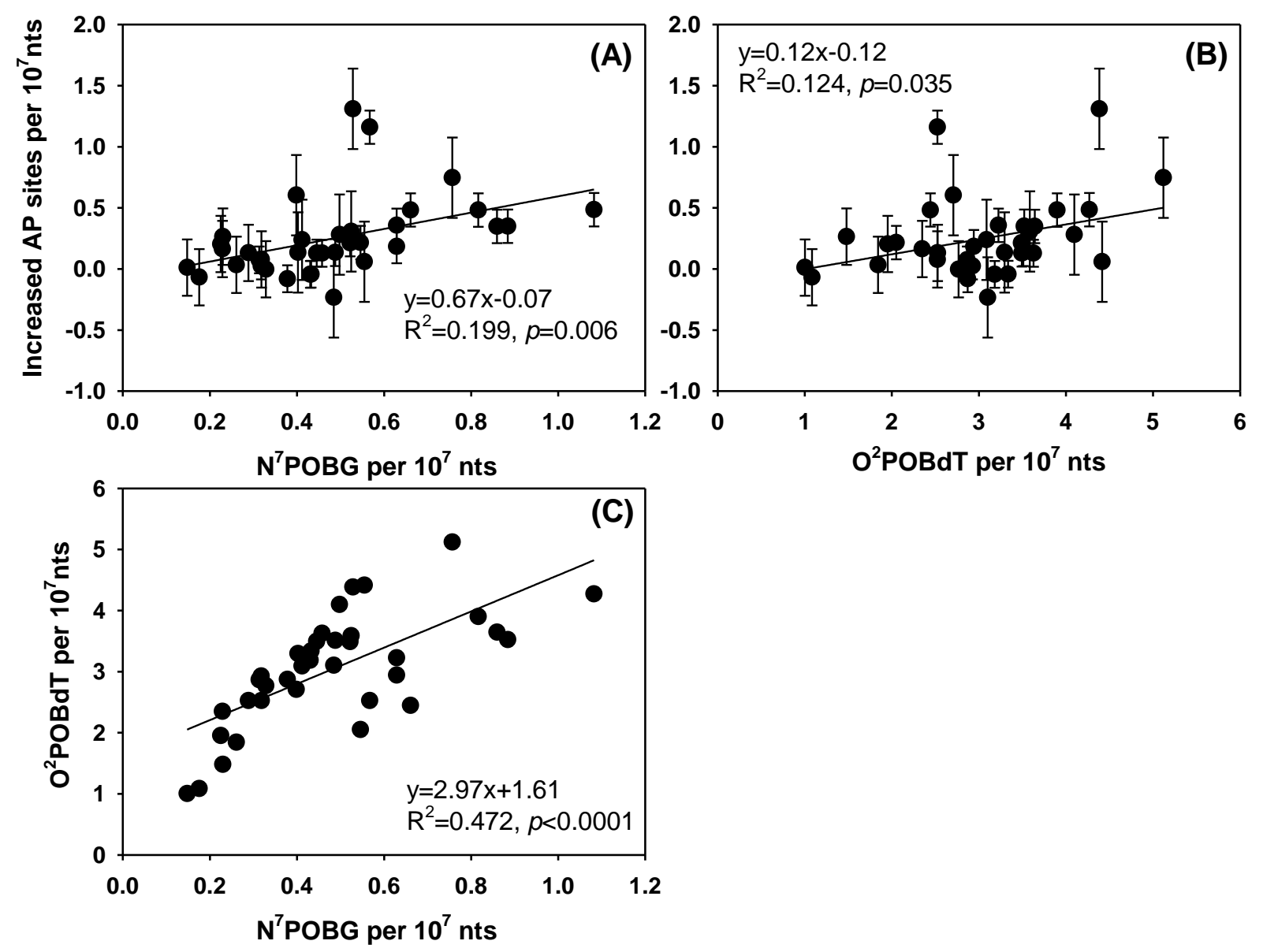

Figure S4. Dependence of increased AP site formation (exposures minus average controls at $10,30,50$ and 70 weeks) on $\mathrm{N}^{7} \mathrm{POBG}(\mathrm{A})$ or $\mathrm{O}^{2} \mathrm{POBdT}(\mathrm{B})$ and dependence of $\mathrm{O}^{2} \mathrm{POBdT}$ on $N^{7} P O B G(C)$ in the liver of rats treated with $5 \mathrm{ppm}$ NNK in drinking water for 70 weeks. The total doses of rats treated with $5 \mathrm{ppm}$ NNK in their drinking water for $10,30,50$ or 70 weeks are 19, 44, 68 and $97 \mathrm{mg} / \mathrm{kg} \mathrm{NNK}$, respectively (2). 


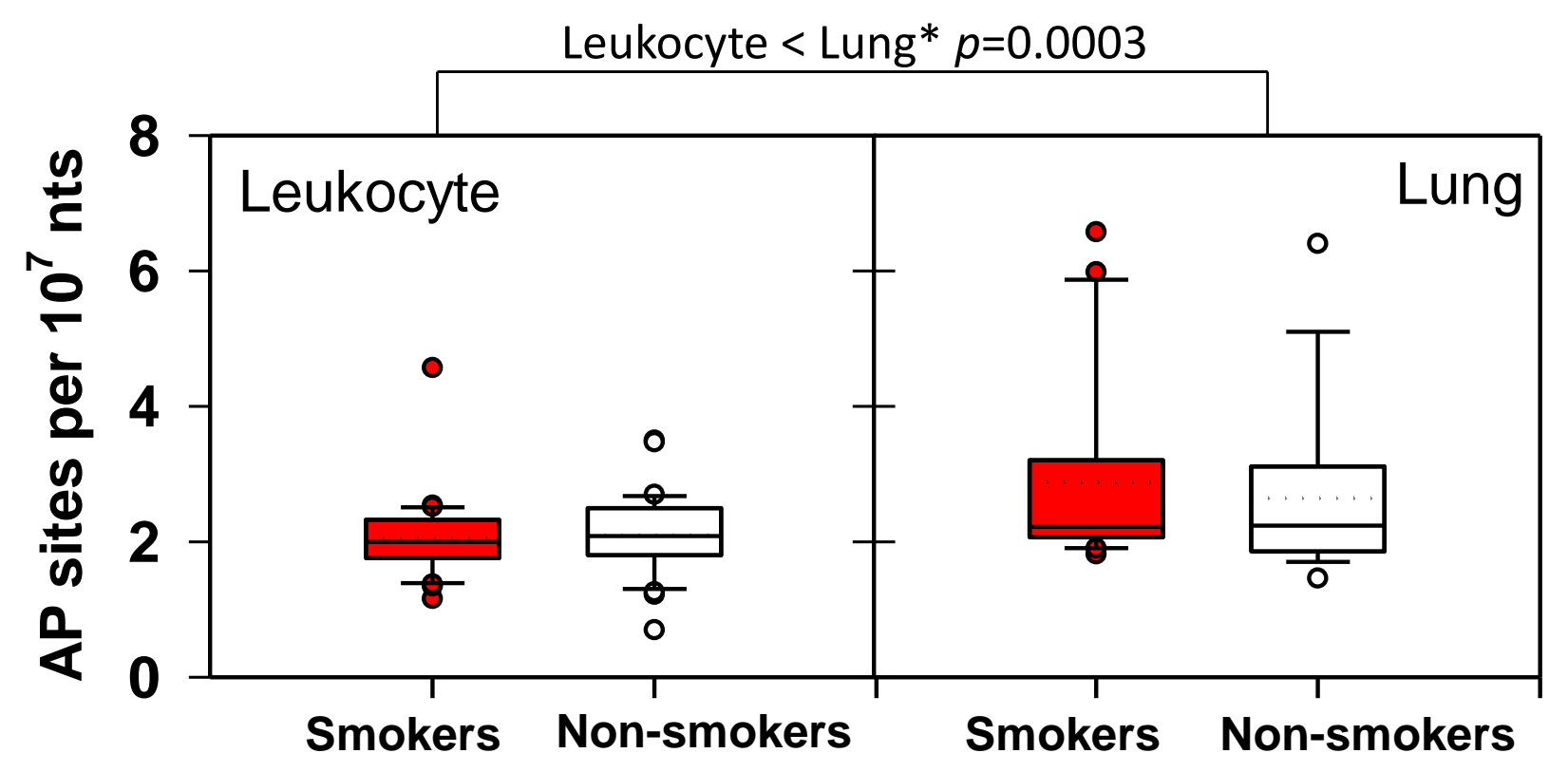

Figure S5. Box and whisker plots of AP site formation in human lung and leukocyte DNA. In lung, $\mathrm{N}=20$ for smokers, $\mathrm{N}=17$ for nonsmokers; in leukocyte, $\mathrm{N}=31$ for both smokers and nonsmokers. There was no significant difference in AP sites between smokers and nonsmokers in both human lung and leukocyte $(p>0.05)$. AP sites in lung are significantly higher than those in leukocyte ( $p=0.0003)$. Shown are the medians (black lines inside the box), the 25th to 75th percentiles (box), the 10th and 90th percentiles (whiskers), the minimum and maximum values (circles), and the averages (dashed lines inside the box). 


\section{REFERENCES}

1. Wang, M., Cheng, G., Villalta, P.W. and Hecht, S.S. (2007) Development of liquid chromatography electrospray ionization tandem mass spectrometry methods for analysis of DNA adducts of formaldehyde and their application to rats treated with $\mathrm{N}$-nitrosodimethylamine or 4(methylnitrosamino)-1-(3-pyridyl)-1-butanone. Chem. Res. Toxicol., 20, 1141-1148.

2. Balbo, S., Johnson, C.S., Kovi, R.C., James-Yi, S.A., O'Sullivan, M.G., Wang, M., Le, C.T., Khariwala, S.S., Upadhyaya, P. and Hecht, S.S. (2014) Carcinogenicity and DNA adduct formation of 4-(methylnitrosamino)-1-(3-pyridyl)-1-butanone and enantiomers of its metabolite 4(methylnitrosamino)-1-(3-pyridyl)-1-butanol in F-344 rats. Carcinogenesis, 35, 2798-2806.

3. Lao, Y., Yu, N., Kassie, F., Villalta, P.W. and Hecht, S.S. (2007) Formation and accumulation of pyridyloxobutyl DNA adducts in F344 rats chronically treated with 4-(methylnitrosamino)-1-(3pyridyl)-1-butanone and enantiomers of its metabolite, 4-(methylnitrosamino)-1-(3-pyridyl)-1butanol. Chem. Res. Toxicol., 20, 235-245.

4. Yang, J., Villalta, P.W., Upadhyaya, P. and Hecht, S.S. (2016) Analysis of O6-[4-(3-pyridyl)-4oxobut-1-yl]-2'-deoxyguanosine and other DNA adducts in rats treated with enantiomeric or racemic N'-nitrosonornicotine. Chem. Res. Toxicol., 29, 87-95.

5. Zhao, L., Balbo, S., Wang, M., Upadhyaya, P., Khariwala, S.S., Villalta, P.W. and Hecht, S.S. (2013) Quantitation of pyridyloxobutyl-DNA adducts in tissues of rats treated chronically with (R)or (S)-N'-nitrosonornicotine (NNN) in a carcinogenicity study. Chem. Res. Toxicol., 26, 15261535.

6. Yang, J., Balbo, S., Villalta, P.W. and Hecht, S.S. (2019) Analysis of acrolein-derived 1,N2propanodeoxyguanosine adducts in human lung DNA from smokers and nonsmokers. Chem. Res. Toxicol., 32, 318-325.

7. Ma, B., Villalta, P.W., Hochalter, J.B., Stepanov, I. and Hecht, S.S. (2019) Methyl DNA phosphate adduct formation in lung tumor tissue and adjacent normal tissue of lung cancer patients. Carcinogenesis, 40, 1387-1394.

8. Villalta, P.W., Hochalter, J.B. and Hecht, S.S. (2017) Ultrasensitive high-resolution mass spectrometric analysis of a DNA adduct of the carcinogen benzo[a]pyrene in human lung. Anal. Chem., 89, 12735-12742.

9. Zhang, S., Balbo, S., Wang, M. and Hecht, S.S. (2011) Analysis of acrolein-derived 1, $\mathrm{N}^{2}-$ propanodeoxyguanosine adducts in human leukocyte DNA from smokers and nonsmokers. Chem. Res. Toxicol., 24, 119-124.

10. Cheng, G., Reisinger, S.A., Shields, P.G., Hatsukami, D.K., Balbo, S. and Hecht, S.S. (2020) Quantitation by liquid chromatography-nanoelectrospray ionization-high resolution tandem mass spectrometry of DNA adducts derived from methyl glyoxal and carboxyethylating agents in leukocytes of smokers and nonsmokers. Chem. Biol. Interact., 327, 109140.

11. Cheng, G., Wang, M., Villalta, P.W. and Hecht, S.S. (2010) Detection of 7-(2'carboxyethyl)guanine but not 7-carboxymethylguanine in human liver DNA. Chem. Res. Toxicol., 23, 1089-1096.

12. Wang, M., Cheng, G., Balbo, S., Carmella, S.G., Villalta, P.W. and Hecht, S.S. (2009) Clear differences in levels of a formaldehyde-DNA adduct in leukocytes of smokers and nonsmokers. Cancer Res., 69, 7170-7174.

13. Balbo, S., Villalta, P.W. and Hecht, S.S. (2011) Quantitation of 7-ethylguanine in leukocyte DNA from smokers and nonsmokers by liquid chromatography-nanoelectrospray-high resolution tandem mass spectrometry. Chem. Res. Toxicol., 24, 1729-1734. 